\title{
Conditions for the stability of switched systems containing unstable subsystems
}

\author{
Yue-E Wang, Hamid Reza Karimi, and Di Wu
}

\begin{abstract}
This paper studies the stability of switched systems in which all the subsystems may be unstable. In addition, some of the switching behaviors of the systems are destabilizing. By using the piecewise Lyapunov function method and taking a tradeoff between the increasing scale and the decreasing scale of the Lyapunov function at switching times, the maximum dwell time for admissible switching signals is obtained and the extended stability results for switched systems in a nonlinear setting are first derived. Then, based on the discretized Lyapunov function method, the switching stabilization problem for linear context is solved. By contrasting with the contributions available in the literature, we do not require that all the switching behaviors of the switching system under consideration are stabilizing. More specifically, even if all the subsystems governing the continuous dynamics are not stable and some of the switching behaviors are destabilizing, the stability of the switched system can still be retained. A numerical example is given to illustrate the validity of the proposed results.
\end{abstract}

Index Terms-Switched nonlinear systems, unstable subsystems, destabilizing, Lyapunov function.

\section{INTRODUCTION}

$\mathbf{S}^{\mathrm{s}}$ WITCHED systems, as an important class of hybrid systems, are composed of a set of continuous-time or discrete-time subsystems and a switching rule acted among subsystems. Over the past two decades, topics involving switched systems have received increasing interest due to the potential applications in many practical systems such as direct injection stratified charge engines [1] and cellular mobile communication systems [2]. For instance, in a cellular mobile communication system, when the user moves from a base station coverage area to another base station coverage area, the previous occupied channel is immediately switched to the pool of available channel, which can be modeled as a switched system. A lot of efforts have been focused on the stability analysis and the control problem of switched systems [3]-[10]. For example, barrier Lyapunov functions for the output tracking control of constrained switched systems was studied in [11]. Work [12] discussed fault-tolerant control of switched nonlinear systems with structural uncertainties. The survey paper [13] gave an overview about the methods for the

Yue-E Wang is with School of Mathematics and Information Science, Shaanxi Normal University, Xi'an 710119, China e-mail: (baihewye@snnu.edu.cn).

Hamid Reza Karimi is with Department of Mechanical Engineering, Politecnico di Milano, 20156 Milan, Italy e-mail:(hamidreza.karimi@polimi.it)

Di Wu is with School of Control Science and Control Engineering, Dalian University of Technology, Dalian 116024, China e-mail:(diwu@dlut.edu.cn).

This work was supported by National Natural Science Foundation of China under Grants 61403241 and the Fundamental Research Funds for the Central Universities under Grant GK201703009 and DUT17RC(3)098. Corresponding author: Di Wu. stability and the stabilization of switched linear systems. The theoretical results and applications for the stability analysis and stabilization of switched systems have been systematized in [14], to which we refer the reader for research purpose.

On the other hand, the stabilization issue of switched systems with unstable subsystems deservers deep investigation due to its theoretical significance and practical application. In fact, even if a switched system contains unstable subsystems, the stability of it can still be guaranteed as long as the switching signal is properly designed. The paper [15] first studied the topic for switched linear systems with some unstable subsystems by using the average dwell time approach. It was shown that if the average dwell time is chosen sufficiently large and the total activation time of unstable subsystems is relatively small compared with that of stable ones, the stability of the switched systems can be guaranteed. Since then a lot of contributions have been devoted to switched systems subject to unstable subsystems. For instance, asynchronous networked control systems can be modeled by switched systems with both stable and unstable subsystems [16]. To sum up, the main stabilization idea is to keep the stability of the whole switched system through the trade-off between the stable subsystems and the unstable ones [17].

However, when all the subsystems are unstable, the stabilization problem is much more challenging since the trade-off idea obviously fails. To solve this issue, the state-dependent switching strategies such as the min-projection strategy and the largest region function strategy have been proposed. Until recently, the dwell time switching strategy was resorted to discuss the stability of switched linear systems with all unstable subsystems under time-dependent switching. In a word, the idea of these methods is to find a switching signal that renders the switched system stable. Switching stabilization has a wide range of applications in fields like hovercraft [18] and electrochromic films [19] and has been employed in various studies. For example, the input-to-state stability of switched delay systems with all unstable subsystems were retained, provided that the switching signal satisfies a dwell-time upper bound condition in [20]. Work [21] extended the results in [20] into an enlarged class of hybrid delayed systems. In [22], stabilization of switched linear systems composed fully of unstable subsystems under dwell time switching was investigated. It is worth pointing out that all the switching behaviors are required to be stabilizing in the above literatures. In fact, even if some of the switching behaviors are destabilizing, one may exploit the good characteristic of the switching behaviors to make the switched system asymptotically stable. These remarks motivate the present work. 
The main objective of this paper is to propose the stabilization conditions of switched systems. The extended stability results for switched systems in a nonlinear setting are first derived by using the piecewise Lyapunov function method and taking a tradeoff between the increasing scale and the decreasing scale of the Lyapunov function at switching times. Then, based on discretized Lyapunov method, the switching stabilization problem for linear context is solved. By contrasting with the results available in the literatures, the contribution of this paper lies in two aspects. First, by further allowing the Lyapunov function to increase during the running time of the active subsystem, no subsystem is required to be stable. Second, it is shown that, even if all the subsystems governing the continuous dynamics are not stable and some of the switching behaviors of the switched systems are destabilizing, the residual stabilizing switching behaviors can successfully stabilize the whole switched system, provided that there are no overly long time intervals between switchings and the destabilizing switchings do not occur too frequently.

The rest of this paper is divided into four sections. Section 2 introduces some preliminaries while Section 3 states our main results regarding the stabilization conditions of switched linear systems. A numerical example is presented in Section 4 and Section 5 concludes the paper.

Notations. $\mathscr{S}=\{1,2, . ., s\} . \mathbb{N}$ denotes the set of nonnegative integer numbers and $\mathbb{N}^{+}=\mathbb{N} /\{0\}$. A function $\gamma: \mathbb{R}^{+} \rightarrow \mathbb{R}^{+}$ is said to be of class $K$ if it is continuous, zero at the origin, and strictly increasing. It is of class $K_{\infty}$ if it is of class $K$ and unbounded. A function $\beta: \mathbb{R}^{+} \times \mathbb{R}^{+} \rightarrow \mathbb{R}^{+}$is of class $K L$ if $\beta(\cdot, t)$ is of class $K$ for each $t \geq 0$ and $\beta(s, \cdot)$ is decreasing to zero for each $s \geq 0$.

\section{PRELIMINARIES}

Consider the switched nonlinear system

$$
\dot{x}(t)=f_{\sigma(t)}(x(t)),
$$

where $x(t) \in \mathbb{R}^{n_{x}}$ is the state; $x\left(t_{0}\right)=x_{0}$ is the initial state; $\sigma(t):[0, \infty) \rightarrow \mathscr{S}$ is a switching signal. It has the switching sequence $\left\{\left(\ell_{0}, t_{0}\right), \ldots,\left(\ell_{k}, t_{k}\right), \ldots, \mid \ell_{k} \in \mathscr{S}, k \in \mathbb{N}\right\}$, which means that the $\ell_{k}$ th subsystem is active when $t \in\left[t_{k}, t_{k+1}\right)$. For $\forall i \in \mathscr{S}$, $f_{i}: \mathbb{R}^{n_{x}} \rightarrow \mathbb{R}^{n_{x}}$ are smooth functions with $f_{i}(0)=0$, and the origin is not a stable equilibrium for any modes $i \in \mathscr{S}$. We assume that no jump occurs in the state at a switching time and that only finitely many switchings can occur in any finite interval. For the sake of clarity, we recall the definition of asymptotic stability for switched systems.

Definition 1.[10] System (1) with switching signal $\sigma(t)$ is said to be asymptotically stable if there exists function $\beta \in$ $K L$, such that for any initial condition $x_{0} \in \mathbb{R}^{n_{x}}$, the solution of system (1) exists over $\mathbb{R}^{+}$and satisfies $|x(t)| \leq \beta\left(\left|x_{0}\right|, t-\right.$ $\left.t_{0}\right), t \geq t_{0} \geq 0$.

In this section, we aim to establish sufficient conditions that guarantee the stability of system (1) when all subsystems of (1) are unstable. In [20], [21], [22], this issue has been studied by requiring that all of the switching behaviors of the switching signal admit stabilizing property to compensate the increment of the multiple Lyapunov functions. In this paper, it is shown that even if some of the switching behaviors play the destabilizing property, the stability of the whole switched system can still be retained. We assume that after $m$ stabilizing switching, the system should undergo a destabilizing switching. Likewise, after $n$ destabilizing switching, the system should undergo a stabilizing switching. For this purpose, we define a new class of switching time sequences. First, we let $p_{l}=m+n, m, n \in \mathbb{N}^{+}$, and define the sets $\Xi_{1}=\{0,1, \ldots, m-1\}$, $\Xi_{2}=\left\{p_{l}-n, \ldots, p_{l}-1\right\}$, and $\Xi=\Xi_{1} \cup \Xi_{2}$. Then, consider a subsequence $\left\{t_{l_{k}}\right\}$ of $\left\{t_{k}\right\}$ with $t_{l_{1}}=t_{1}, t_{l_{0}}=t_{0}=0$, which satisfies $l_{k+1}-l_{k}=p_{l}, l_{k}, k, p_{l} \in \mathbb{N}^{+}$. It is clear that any switching point in $\left[t_{l_{k}}, t_{l_{k+1}}\right)$ can be described as $t_{l_{k}+p}, p \in \Xi$. For any $p \in \Xi, k \in \mathbb{N}$, suppose that $0<\tau_{\min } \leq t_{l_{k}+p+1}-t_{l_{k}+p} \leq \tau_{\max }$.

To investigate the stability property of system (1), we choose the following piecewise Lyapunov functions

$$
V(t)=V_{\sigma(t)}(t, x),
$$

where $V_{i}(t, x): \mathbb{R}^{+} \times \mathbb{R}^{n_{x}} \rightarrow \mathbb{R}^{+}, i \in \mathscr{S}$ are continuous non-negative functions, and define the notations $V_{i}\left(t_{k}^{+}\right)=$ $\lim _{t \rightarrow t_{k}^{+}} V_{i}(t)$ and $V_{i}\left(t_{k}^{-}\right)=\lim _{t \rightarrow t_{k}^{-}} V_{i}(t)$. Now, we are ready to establish the stability result for system (1).

Theorem 1. Consider switched nonlinear system (1) with switching signal $\sigma(t)$. If there exists a piecewise continuous Lyapunov function defined in (2) such that

(i) for each $i \in \mathscr{S}$, there exist functions $\alpha_{j} \in K_{\infty}(j=1,2)$ and a constant $\eta>0$ such that, for all $x \in \mathbb{R}^{n_{x}}$ and $t \geq t_{0} \geq 0$,

$$
\begin{aligned}
& \alpha_{1}(|x|) \leq V_{i}(t, x) \leq \alpha_{2}(|x|), \\
& \dot{V}_{i}(t) \leq \eta V_{i}(t)
\end{aligned}
$$

(ii) there are two constants $0<\rho<1, \mu \geq 1$ such that for all $i \neq j, i, j \in \mathscr{S}, k \in \mathbb{N}^{+}$,

$$
V_{i}\left(t_{l_{k}+p}^{+}\right) \leq \begin{cases}\rho V_{j}\left(t_{l_{k}+p}^{-}\right), & p \in \Xi_{1} \\ \mu V_{j}\left(t_{l_{k}+p}^{-}\right), & p \in \Xi_{2},\end{cases}
$$

(iii) $\Gamma_{1}<1$ and $\frac{m}{n}>\frac{\ln \Gamma_{2}}{-\ln \Gamma_{1}}$, where, $\Gamma_{1}=\rho M, \Gamma_{2}=\mu M, M=e^{\eta \tau_{\max }}$. Then, switched nonlinear system (1) with switching signal $\sigma(t)$ is asymptotically stable.

Proof. See the Appendix.

Corollary 1. Consider switched system (1) with switching signal $\sigma(t)$. If there exists a piecewise continuous Lyapunov function defined in (2) such that

(i)' for each $i \in \mathscr{S}$, there exist functions $\alpha_{j} \in K_{\infty}(j=1,2)$ and a constant $\eta>0$ such that, for all $x \in \mathbb{R}^{n_{x}}$ and $t \geq t_{0} \geq 0$, $\alpha_{1}(|x|) \leq V_{i}(t, x) \leq \alpha_{2}(|x|)$ and $\dot{V}_{i}(t) \leq \eta V_{i}(t)$,

(ii)' there is a constant $0<\rho<1$ such that for all $i \neq j, i, j \in$ $\mathscr{S}, V_{i}\left(t_{k}^{+}\right) \leq \rho V_{j}\left(t_{k}^{-}\right), k \in \mathbb{N}^{+}$,

(iii)' $\rho e^{\eta \tau_{\max }}<1$.

Then, switched system (1) with switching signal $\sigma(t)$ is asymptotically stable.

Remark 1. The results in [23] apply to cases where some subsystems of the switched systems are not necessarily stable under the influence of asynchronous switching or input delay. The method in the literature fail to deal with the stability of system (1) in the paper because all the subsystems are allowed to be unstable. 
Remark 2. In some previous results for switched nonlinear systems with all unstable subsystems [20], [21], [22], the switching behaviors are required to decrease the value of the piecewise continuous Lyapunov functions at switching times. However, this viewpoint is too strict since the destabilizing property of some of switching behaviors is not considered. In Theorem 1, even if all the subsystems are unstable and some of the switching behaviors are destabilizing, the residual stabilizing switching behaviors can successfully stabilize the switched system.

\section{STABILIZATION FOR SWITCHED LINEAR SYSTEM}

Consider the switched linear system

$$
\dot{x}(t)=A_{\sigma(t)} x(t),
$$

where $x(t)$ and $\sigma(t)$ are described the same as in (1). All the subsystem matrices $A_{i}, i \in \mathscr{S}$ are supposed to have eigenvalues located in the right half-plane. Without loss of generality, we suppose that the subsystems $i\left(i \in \mathscr{S}^{-}=\{1,2, \ldots, r\}, 1 \leq\right.$ $r<s, r \in \mathbb{N})$ are activated in $\left[t_{l_{k}+p}, t_{l_{k}+p+1}\right), k \in \mathbb{N}^{+}, p \in \Xi_{1}$, while the other subsystems $j\left(j \in \mathscr{S}^{+}=\{r+1, r+2, \ldots, s\}\right)$ are activated in $\left[t_{l_{k}+p}, t_{l_{k}+p+1}\right), k \in \mathbb{N}^{+}, p \in \Xi_{2}$ and introduce the set $S=\{0,1,2, \ldots, L-1\}$ with the integer $L \geq 1$.

Theorem 2. Given scalars $\eta>0,0<\rho<1, \mu \geq 1$, consider switched system (6), if the following conditions hold:

i) for each $i \in \mathscr{S}^{-}$, there exist matrices $P_{i}^{q}>0, q=0,1, \ldots, L$, such that for all $i, j \in \mathscr{S}^{-}, i \neq j$,

$$
\begin{array}{r}
A_{i}^{T} P_{i}^{q}+P_{i}^{q} A_{i}+\varpi_{i}^{q}-\eta P_{i}^{q}<0, q \in S, \\
A_{i}^{T} P_{i}^{q+1}+P_{i}^{q+1} A_{i}+\varpi_{i}^{q}-\eta P_{i}^{q+1}<0, q \in S, \\
A_{i}^{T} P_{i}^{L}+P_{i}^{L} A_{i}-\eta P_{i}^{L}<0, \\
P_{i}^{0}-\rho P_{j}^{L} \leq 0,
\end{array}
$$

ii) for each $i \in \mathscr{S}^{+}$, there exist matrices $Q_{i}>0$, such that for all $i, j \in \mathscr{S}^{+}, i \neq j$,

$$
A_{i}^{T} Q_{i}+Q_{i} A_{i}-\eta Q_{i}<0 \quad \text { and } \quad Q_{i}-\mu Q_{j} \leq 0,
$$

iii) for all $i \in \mathscr{S}^{-}, j \in \mathscr{S}^{+}$,

$$
P_{i}^{0}-\rho Q_{j} \leq 0 \quad \text { and } \quad Q_{j}-\mu P_{i}^{L} \leq 0,
$$

iv) $\Gamma_{1}<1$ and $\frac{m}{n}>\frac{\ln \Gamma_{2}}{-\ln \Gamma_{1}}$,

where, $\Gamma_{1}=\rho M, \Gamma_{2}=\mu M, M=e^{\eta \tau_{\max }},{\varpi_{i}^{(q)}}^{(q)} L\left(P_{i}^{q+1}-\right.$ $\left.P_{i}^{q}\right) / \tau_{\min }$. Then, switched linear system (6) is asymptotically stable.

Proof. See the Appendix.

\section{NUMERICAL EXAMPLE}

In this section, we present one example to illustrate the effectiveness of the proposed method.

Example 1. Most electronic systems, mechanical systems, and aeroengine control systems can be modeled as multiintegrator systems [24]. For simplicity, we consider a given double integrator system as the example and first design a suitable feedback controller for it. Considering the scenario as following, the controller may have several backup controllers, but all of controllers are partly failure or totally failure. The double integrator system will be unstable with any controller. However, we can design a switching law to stabilize the double integrator system.

Consider the double integrator system with the state matrix $A=\left[\begin{array}{ll}0 & 1 \\ 0 & 0\end{array}\right]$, the input matrix $B=\left[\begin{array}{l}0 \\ 1\end{array}\right]$, and the feedback control law $K=\left[\begin{array}{ll}-1 & -2\end{array}\right]$. The controller and the backup controllers are partly failure or totally failure and they can be given as $K_{1}=\left[\begin{array}{ll}0 & 0\end{array}\right], K_{2}=\left[\begin{array}{ll}-1 & 0\end{array}\right]$, and $K_{3}=\left[\begin{array}{ll}0 & -2\end{array}\right]$, which result in the closed-loop system as

$$
\dot{x}(t)=A_{\sigma(t)} x(t), \mathscr{S}=\{1,2,3\}
$$

with

$$
A_{1}=\left[\begin{array}{ll}
0 & 1 \\
0 & 0
\end{array}\right], A_{2}=\left[\begin{array}{cc}
0 & 1 \\
-1 & 0
\end{array}\right], A_{3}=\left[\begin{array}{cc}
0 & 1 \\
0 & -2
\end{array}\right] .
$$

Obviously, we can check that all of matrixes are unstable.

Choose a switching sequence that satisfies $t_{n+1}-t_{n}=0.12 \mathrm{~s}$, $n \in \mathbb{N}$. we see that $\tau_{\min }=\tau_{\max }=0.12 s$. For, $L=8.333$, $\rho=0.76, \mu=1.3$, and $\eta=1.9$, the state trajectory and the evolution of the Lyapunov function under the switching signal $\sigma$ are shown in Figs. 1 and 2, respectively.

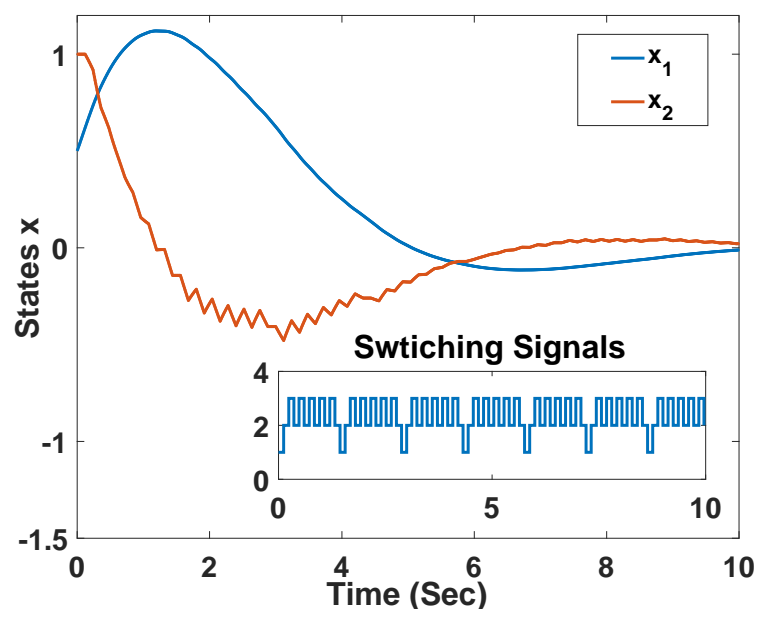

Fig. 1. State trajectories of the switched system (13)

\section{CONCLUSION}

We have studied the stability of switched systems in which all the subsystems may be unstable and some of the switching behaviors are destabilizing. By using the piecewise Lyapunov function method and taking a tradeoff between the increasing and the decreasing scales of the Lyapunov function at switching times, the extended stability results for switched systems in a nonlinear setting have been derived. Then, based on the discretized Lyapunov function method, the switching stabilization problem for linear context has been solved.

\section{APPENDIX}

Proof of Theorem 1. Set $\Gamma=\Gamma_{1}^{m} \Gamma_{2}^{n}$. We use the mathematical induction method to prove that for $\forall t \in\left[t_{k}+p, t_{l_{k}+p+1}\right), k \in$ 


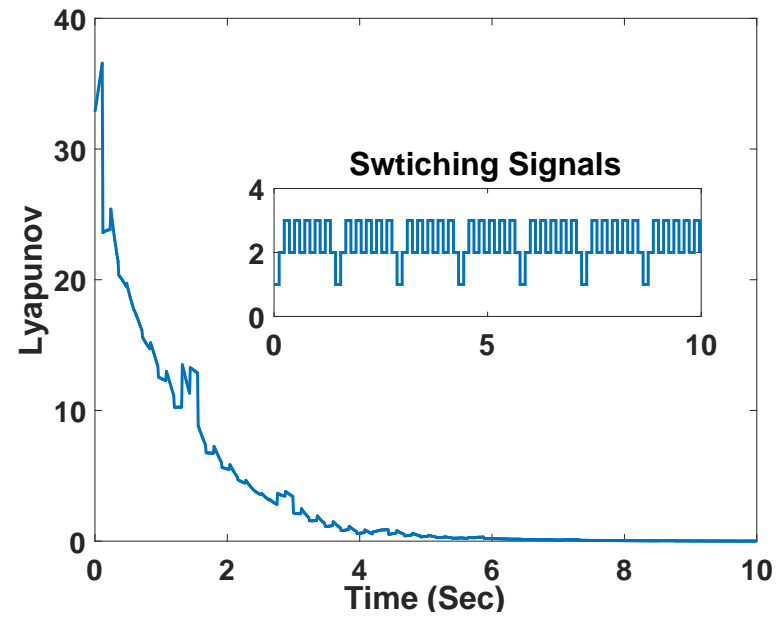

Fig. 2. Evolution of Lyapunov function.

$\mathbb{N}^{+}$,

$$
\begin{aligned}
& \text { if } p \in \Xi_{1}, V_{\sigma\left(t_{l_{k}+p}\right)}(t) \leq \Gamma_{1}^{p+1} \Gamma^{k-1} M V_{\sigma\left(t_{0}\right)}\left(t_{0}\right), \\
& \text { if } p \in \Xi_{2}, V_{\sigma\left(t_{l_{k}+p}\right)}(t) \leq \Gamma_{2}^{p+1-m} \Gamma_{1}^{m} \Gamma^{k-1} M V_{\sigma\left(t_{0}\right)}\left(t_{0}\right)
\end{aligned}
$$

From condition (ii), we note for later use that, for $\forall t \in$ $\left[t_{l_{k}+p}, t_{l_{k}+p+1}\right), k \in \mathbb{N}^{+}$,

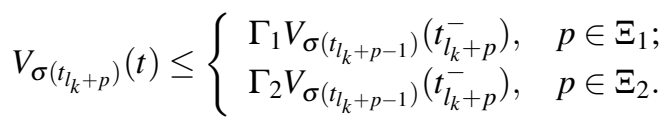

Now, look at the case of $\left[t_{l_{1}+p}, t_{l_{1}+p+1}\right), p \in \Xi$. When $p=0$, that is just $\left[t_{l_{1}}, t_{l_{1}+1}\right)$, it follows from (4) that for $\forall t \in\left[t_{0}, t_{l_{1}}\right)$,

$$
\begin{aligned}
V_{\sigma\left(t_{0}\right)}(t) & \leq e^{\eta\left(t-t_{0}\right)} V_{\sigma\left(t_{0}\right)}\left(t_{0}\right) \\
& \leq e^{\eta \tau_{\max }} V_{\sigma\left(t_{0}\right)}\left(t_{0}\right) \\
& =M V_{\sigma\left(t_{0}\right)}\left(t_{0}\right),
\end{aligned}
$$

which clearly gives that

$$
V_{\sigma\left(t_{0}\right)}\left(t_{l_{1}}^{-}\right) \leq M V_{\sigma\left(t_{0}\right)}\left(t_{0}\right) .
$$

Then, combining (16) and (18) leads to, when $t \in\left[t_{l_{1}}, t_{l_{1}+1}\right)$, $V_{\sigma\left(t_{l_{1}}\right)}(t) \leq \Gamma_{1} M V_{\sigma\left(t_{0}\right)}\left(t_{0}\right)$.

Next, suppose that for $t \in\left[t_{l_{1}+p-1}, t_{l_{1}+p}\right), p-1 \in \Xi_{1}$, (14) holds. Thus, when $t \in\left[t_{l_{1}+p}, t_{l_{1}+p+1}\right), p \in \Xi_{1}$, using (14) and (16), we deduce that

$$
\begin{aligned}
V_{\sigma\left(t_{l_{1}+p}\right)}(t) & \leq \Gamma_{1} V_{\sigma\left(t_{l_{1}+p-1}\right)}\left(t_{l_{1}+p}^{-}\right) \\
& \leq \Gamma_{1} \Gamma_{1}^{p} M V_{\sigma\left(t_{0}\right)}\left(t_{0}\right) \\
& =\Gamma_{1}^{p+1} M V_{\sigma\left(t_{0}\right)}\left(t_{0}\right) .
\end{aligned}
$$

Therefore, from (19), when $t \in\left[t_{l_{1}+m-1}, t_{l_{1}+m}\right)$, it holds

$$
V_{\sigma\left(t_{l_{1}+m-1}\right)}(t) \leq \Gamma_{1}^{m} M V_{\sigma\left(t_{0}\right)}\left(t_{0}\right) .
$$

When $t \in\left[t_{l_{1}+m}, t_{l_{1}+m+1}\right)$, with (5) and (20), we get that $V_{\sigma\left(t_{l_{1}+m}\right)}(t) \leq M \mu \Gamma_{1}^{m} M V_{\sigma\left(t_{0}\right)}\left(t_{0}\right)=\Gamma_{2} \Gamma_{1}^{m} M V_{\sigma\left(t_{0}\right)}\left(t_{0}\right)$. Next, suppose that for $t \in\left[t_{l_{1}+p-1}, t_{l_{1}+p}\right), p-1 \in \Xi_{2}$, (15) holds. Thus, when $t \in\left[t_{l_{1}+p}, t_{l_{1}+p+1}\right), p \in \Xi_{2}$, from (15) and (16), one has $V_{\sigma\left(t_{l_{1}+p}\right)}(t) \leq \Gamma_{2} V_{\sigma\left(t_{l_{1}+p-1}\right)}\left(t_{l_{1}+p}^{-}\right) \leq \Gamma_{2}^{p+1-m} \Gamma_{1}^{m} M V_{\sigma\left(t_{0}\right)}\left(t_{0}\right)$.
Suppose that for all $t \in\left[t_{l_{k-1}+p}, t_{l_{k-1}+p+1}\right)$, if $p \in \Xi_{1}$, (14) holds, if $p \in \Xi_{2}$, (15) holds. We will show that for any $t \in$ $\left[t_{l_{k}+p}, t_{l_{k}+p+1}\right)$, if $p \in \Xi_{1}$, (14) holds, and if $p \in \Xi_{2}$, (15) holds. First, we show the case of $t \in\left[t_{l_{k}}, t_{l_{k}+1}\right)$, i.e. $p=0$. From (16),

$$
V_{\sigma\left(t_{l_{k}}\right)}(t) \leq \Gamma_{1} V_{\sigma\left(t_{l_{k}-1}\right)}\left(t_{l_{k}}^{-}\right) \leq \Gamma_{1} \Gamma^{k-1} M V_{\sigma\left(t_{0}\right)}\left(t_{0}\right) .
$$

Then, suppose that for $t \in\left[t_{l_{k}+p-1}, t_{l_{k}+p}\right), p-1 \in \Xi_{1}$, (14) holds. By using (14) and (21), it can be easily shown that (14) holds for any $t \in\left[t_{l_{k}+p}, t_{l_{k}+p+1}\right), p \in \Xi_{1}$. Thus, taking $p=m$, we can show that (15) holds for $t \in$ $\left[t_{l_{k}+m}, t_{l_{k}+m+1}\right)$. Next, suppose that for any $t \in\left[t_{l_{k}+p-1}, t_{l_{k}+p}\right)$, $p-1 \in \Xi_{2}$, (15) holds. Then, considering (15) and (16), for $t \in\left[t_{l_{k}+p}, t_{l_{k}+p+1}\right), p \in \Xi_{2}$, it is true that $V_{\sigma\left(t_{l_{k}+p}\right)}(t) \leq$ $\Gamma_{2} V_{\sigma\left(t_{k}+p-1\right)}\left(t_{l_{k}+p}^{-}\right) \leq \Gamma_{2}^{p+1-m} \Gamma_{1}^{m} \Gamma^{k-1} M V_{\sigma\left(t_{0}\right)}\left(t_{0}\right)$.

When $t \in\left[t_{l_{k}+p}, t_{l_{k}+p+1}\right), k \in \mathbb{N}^{+}, p \in \Xi, t-t_{1} \leq k(m+$ n) $\tau_{\max }$, which implies

$$
k \geq \frac{t-t_{1}}{(m+n) \tau_{\max }} \geq \frac{t-t_{0}-\tau_{\max }}{(m+n) \tau_{\max }} .
$$

The inequalities $\Gamma_{1}<1$ and $\frac{m}{n}>\frac{\ln \Gamma_{2}}{-\ln \Gamma_{1}}$ in condition (iii) yield that

$$
\Gamma_{1}^{p+1}<1 \quad \text { and } \quad \Gamma_{2}^{p+1-m} \Gamma_{1}^{m} \leq \Gamma_{2}^{n} \Gamma_{1}^{m}<1 .
$$

From (23), the estimates (14) and (15) easily give that, when $t \in\left[t_{l_{k}+p}, t_{l_{k}+p+1}\right), k \in \mathbb{N}^{+}, p \in \Xi$,

$$
V_{\sigma\left(t_{k}+p\right)}(t) \leq \Gamma^{k-1} M V_{\sigma\left(t_{0}\right)}\left(t_{0}\right)
$$

Considering (3) and (24), when $t \in\left[t_{l_{k}+p}, t_{l_{k}+p+1}\right), k \in \mathbb{N}^{+}$, $p \in \Xi$, it holds that

$$
\begin{aligned}
|x(t)| & \leq \alpha_{1}^{-1}\left(\Gamma^{k-1} M V\left(t_{0}\right)\right) \\
& \leq \alpha_{1}^{-1}\left(\Gamma^{\frac{t-t_{0}-\tau_{\max }}{(m+n) \tau_{\max }}-1} M V\left(t_{0}\right)\right) \\
& \leq \alpha_{1}^{-1}\left(\Gamma^{\frac{t-t_{0}-\tau_{\max }}{(m+n) \tau_{\max }}-1} M \alpha_{2}\left(\left|x_{0}\right|\right)\right) .
\end{aligned}
$$

In view of condition $\Gamma<1$ and $\alpha_{1}, \alpha_{2} \in K_{\infty}$, it is clear that switched system (1) is asymptotically stable.

Proof of Theorem 2. We construct the multiple linear copositive Lyapunov function $V(t)=V_{\sigma}(t)$ in the quadratic form of $V_{i}(t)=x^{T}(t) P_{i}(t) x(t), i \in \mathscr{S}^{-}$and $V_{i}(t)=x^{T}(t) Q_{i} x(t), i \in \mathscr{S}^{+}$ where $Q_{i}, i \in \mathscr{S}^{+}$is positive definite matrix and $P_{i}(t), i \in \mathscr{S}^{-}$ is time-scheduled positive definite matrix. In order to render the stability condition numerically computable, we divide the interval $\mathscr{N}_{k p}=\left[t_{l_{k}+p}, t_{l_{k}+p}+\tau_{\min }\right), p \in \Xi_{1}$ into $L$ segments $\mathscr{N}_{k p}^{q}=\left[t_{l_{k}+p}+\theta_{q}, t_{l_{k}+p}+\theta_{q+1}\right), q \in S$ of equal length $d=$ $\tau_{\min } / L$, and then $\theta_{q}=q d=q \tau_{\min } / L, q \in S$. The continuous matrix function $P_{i}(t), t \in\left[t_{l_{k}+p}, t_{l_{k}+p+1}\right)$ is chosen to be linear within each segment $\mathscr{N}_{k p}^{q}, q \in S$. Let $P_{i}^{q}=P_{i}\left(t_{l_{k}+p}+\theta_{q}\right)$, then since the matrix function $P_{i}(t), i \in \mathscr{S}^{-}$is piecewise linear in $\left[t_{l_{k}+p}, t_{l_{k}+p}+\tau_{\min }\right)$, it can be expressed in terms of the values at segmentation points using a linear interpolation formula, i.e., for $0 \leq \alpha \leq 1, q \in S$, and $t \in \mathscr{N}_{k p}^{q}$,

$$
P_{i}(t)=P_{i}\left(t_{l_{k}+p}+\theta_{q}+\alpha d\right)=(1-\alpha) P_{i}^{q}+\alpha P_{i}^{q+1}=P_{i}^{(q)}(\alpha)
$$


where $\alpha=\left(t-t_{l_{k}+p}-\theta_{q}\right) / d$.

Then, the continuous matrix function $P_{i}(t), i \in \mathscr{S}$ is completely determined by $P_{i}^{q}, q \in S \bigcup\{L\}, i \in \mathscr{S}^{-}$in the minimal dwell time interval $\left[t_{l_{k}+p}, t_{l_{k}+p}+\tau_{\min }\right)$. In the interval $\left[t_{l_{k}+p}+\tau_{\min }, t_{l_{k}+p+1}\right)$, the matrix function $P_{i}(t), i \in \mathscr{S}^{-}$is chosen as $P_{i}(t)=P_{i}^{L}, i \in \mathscr{S}$. To sum up, the discretized matrix function $P_{i}(t), i \in \mathscr{S}^{-}$is expressed as

$$
P_{i}(t)= \begin{cases}P_{i}^{(q)}(\alpha), & t \in \mathscr{N}_{k p}^{q}, q \in S \\ P_{i}^{L}, & t \in\left[t_{l_{k}+p}+\tau_{\min }, t_{l_{k}+p+1}\right) .\end{cases}
$$

and the corresponding discretized Lyapunov function for the $i$ th $\left(i \in \mathscr{S}^{-}\right)$mode is $V_{i}(t)=x^{T}(t) P_{i}^{(q)}(\alpha) x(t), t \in \mathscr{N}_{k p}^{q}, q \in$ $S$, and $V_{i}(t)=x^{T}(t) P_{i}^{L} x(t), t \in\left[t_{l_{k}+p}+\tau_{\min }, t_{l_{k}+p+1}\right)$. One can prove easily that (3) in condition (i) of Theorem 1 is satisfied.

For $t \in \mathscr{N}_{k p}^{q}$, from (26), it follows that

$$
\begin{aligned}
x^{T}(t) \dot{P}_{i}(t) x(t) & =x^{T}(t) \frac{L\left(P_{i}^{q+1}-P_{i}^{q}\right)}{\tau_{\min }} x(t) \\
& =x^{T}(t) \varpi_{i}^{(q)} x(t) \\
\text { and } \quad \dot{x}^{T}(t) P_{i}(t) x(t) & =x^{T}(t) A_{i}^{T} P_{i}^{(q)}(\alpha) x(t) .
\end{aligned}
$$

Therefore, from (28) and (29), we have, for any $t \in \mathscr{N}_{k p}^{q}$,

$$
\begin{aligned}
\dot{V}_{i}(t)-\eta V_{i}(t)= & x^{T}(t) \dot{P}_{i}(t) x(t)+2 \dot{x}^{T}(t) P_{i}(t) x(t) \\
& -\eta x^{T}(t) P_{i}^{(q)}(\alpha) x(t) \\
= & x^{T}(t)\left[A_{i}^{T} P_{i}^{(q)}(\alpha)+P_{i}^{(q)}(\alpha) A_{i}\right. \\
& \left.-\eta P_{i}^{(q)}(\alpha)+\varpi_{i}^{(q)}\right] x(t) .
\end{aligned}
$$

have

Let $\Xi_{i}^{(q)}(\alpha)=A_{i}^{T} P_{i}^{(q)}(\alpha)+P_{i}^{(q)}(\alpha) A_{i}+\bar{\varpi}_{i}^{(q)}$. From (26), we

$$
\begin{aligned}
\Xi_{i}^{(q)}(\alpha)= & A_{i}^{T}\left[(1-\alpha) P_{i}^{q}+\alpha P_{i}^{q+1}\right] \\
& +\left[(1-\alpha) P_{i}^{q}+\alpha P_{i}^{q+1}\right] A_{i}+\varpi_{i}^{(q)} \\
= & (1-\alpha) \Xi_{i, 1}^{(q)}+\alpha \Xi_{i, 2}^{(q)},
\end{aligned}
$$

where $\Xi_{i, 1}^{(q)}=A_{i}^{T} P_{i}^{q}+P_{i}^{q} A_{i}+\varpi_{i}^{(q)}$ and $\Xi_{i, 2}^{(q)}=A_{i}^{T} P_{i}^{q+1}+$ $P_{i}^{q+1} A_{i}+\bar{\varpi}_{i}^{(q)}$.

The inequalities (7), (8), and (26) give that, for any $t \in \mathscr{N}_{k p}^{q}$,

$$
\begin{aligned}
\dot{V}_{i}(t)-\eta V_{i}(t)= & x^{T}(t)(1-\alpha)\left[\Xi_{i, 1}^{(q)}-\eta P_{i}^{q}\right] x(t) \\
& +x^{T}(t) \alpha\left[\Xi_{i, 2}^{(q)}-\eta P_{i}^{q+1}\right] x(t) \\
< & 0 .
\end{aligned}
$$

Moreover, by $P_{i}(t)=P_{i}^{L}$ and (9), when $t \in\left[t_{l_{k}+p}+\right.$ $\left.\tau_{\min }, t_{l_{k}+p+1}\right)$, we can derive $\dot{V}_{i}(t)-\eta V_{i}(t)=x^{T}(t)\left[A_{i}^{T} P_{i}^{L}+\right.$ $\left.P_{i}^{L} A_{i}\right] x(t)-\eta x^{T}(t) P_{i}^{L} x(t)<0$.

On the other hand, for $\forall t \in\left[t_{l_{k}+p}, t_{l_{k}+p+1}\right), p \in \Xi_{2}$, from (11), $\dot{V}_{i}(t)-\eta V_{i}(t)=x^{T}(t)\left[A_{i}^{T} Q_{i}+Q_{i} A_{i}\right] x(t)-\eta x^{T}(t) Q_{i} x(t)<0$.

To summarize, we have, for $\forall t \in\left[t_{l_{k}+p}, t_{l_{k}+p+1}\right), p \in \Xi$, $\dot{V}_{i}(t) \leq \eta V_{i}(t)$. Hence, (4) in condition (i) of Theorem 1 is satisfied. Furthermore, by (10), (12), and (27), we have $V_{i}\left(t_{l_{k}+p}^{+}\right) \leq \rho V_{j}\left(t_{l_{k}+p}^{-}\right), p \in \Xi_{1}$ and $V_{i}\left(t_{l_{k}+p}^{+}\right) \leq \mu V_{j}\left(t_{l_{k}+p}^{-}\right), p \in$ $\Xi_{2}$. that is to say, condition (ii) of Theorem 1 is satisfied.

Therefore, considering iv), the stability of system (6) governed by any switching law $\sigma(t)$ can be established by Theorem 1 .

\section{REFERENCES}

[1] M. Rinehart, M. Dahleh, D. Reed, and I. Kolmanovsky, "Suboptimal control of switched systems with an application to the DISC Engine," IEEE Trans. Control Syst. Technol., vol. 16, no. 2, pp. 189-201, Mar. 2008.

[2] T. S. Rappaport, Wireless communications: principles and practice. New Jersey: prentice hall PTR, 1996.

[3] J. Zhang, H. Zhang, and T. Feng, "Distributed optimal consensus control for nonlinear multiagent system with unknown dynamic," IEEE Trans. Neur. Net. Lear., DOI: 10.1109/TNNLS.2017.2728622, 2018.

[4] X. M. Sun, D. Wu, C. Y. Wen, and W. Wang, " A novel stability analysis for networked predictive control systems," IEEE Trans. Circuits Syst. II Exp. Briefs, vol. 61, no. 6, pp. 453-457, June, 2014.

[5] Z. C. Li, A. Gao, and Y. Zhang, "Exponential stability analysis and stabilization for continuous time-delay systems with controller failure," IEEE Trans. Circuits Syst. II Exp. Briefs, vol. 65, no. 3, pp. 356-360, Mar. 2018.

[6] A. Lekić, and D. Stipanović, "Hysteresis Switching Control of the Ćuk Converter Operating in Discontinuous Conduction Modes," IEEE Trans. Circuits Syst. II Exp. Briefs, vol. 64, no. 9, pp. 1077-1081, Sep. 2017.

[7] D. Liberzon, "Finite data-rate feedback stabilization of switched and hybrid linear systems," Automatica, vol. 50, no. 2, pp. 409-420, Feb. 2014.

[8] Y. E. Wang, X. M. Sun, and B. W. Wu, "Lyapunov-Krasovskii functionals for switched nonlinear input delay systems under asynchronous switching," Automatica, vol. 61, pp. 126-133, Nov. 2015.

[9] J. Zhao and D. J. Hill, "On stability, $L_{2}$-gain and $H_{\infty}$ control for switched systems," Automatica, vol. 44, no. 5, pp. 1220-1232, May 2008.

[10] X. D. Zhao, L. X. Zhang, P. Shi, and M. Liu, "Stability and stabilization of switched linear systems with mode-dependent average dwell time," IEEE Trans. Autom. Control, vol. 57, no. 7, pp. 1809-1815, Jul. 2012.

[11] B. Niu and J. Zhao, "Barrier Lyapunov Functions for the output tracking control of constrained nonlinear switched systems," Syst. Control Lett., vol. 62, no. 10, pp. 963-971, Oct. 2013.

[12] J. Fu, T. Y. Chai, Y. Jin, and C. Y. Su, "Fault-tolerant control of a class of switched nonlinear systems with structural uncertainties," IEEE Trans. Circuits Syst. II Exp. Briefs, vol. 63, no. 2, pp. 201-205, Feb. 2016.

[13] H. Lin and P. J. Antsaklis, "Stability and stabilizability of switched linear systems: a survey of recent results," IEEE Trans. Autom. Control, vol. 54, no. 2, pp. 308-322, Feb. 2009.

[14] D. Liberzon, Switching in systems and control. Birkhauser, Boston, MA, 2003.

[15] G. S. Zhai, B. Hu, K. Yasuda, and A. N. Michel, "Stability analysis of switched systems with stable and unstable subsystems: an average dwell time approach," Int. J. Syst. Sci., vol. 32, no. 8, pp. 1055-1061, Nov. 2010.

[16] D. Ma and J. Zhao, "Stabilization of networked switched linear systems: An asynchronous switching delay system approach," Syst. Control Lett., vol. 77, pp. 46-54, Mar. 2015.

[17] H. Yang, B. Jiang, and V. Cocquempot, Stabilization of switched nonlinear systems with unstable modes. Springer International Publishing, 2014.

[18] K. Tanaka, M. Iwasaki, and H. O. Wang, "Switching control of an R/C hovercraft: stabilization and smooth switching," IEEE Trans. Syst., Man, Cybern. B, Cybern., vol. 31, no. 6, pp. 853-863, Dec. 2001.

[19] D. Chatzikyriakou, N. Krins, B. Gilbert, P. Colson, and J. Dewalque, "Mesoporous amorphous tungsten oxide electrochromic films: a Raman analysis of their good switching behavior," ELectrochim. Acta, vol. 137, pp. 75-82, Aug. 2014.

[20] J. Liu, X. Z. Liu, and W. C. Xie, "Input-to-state stability of impulsive and switching hybrid systems with time-delay," Automatica, vol. 47, no. 5, pp. 899-908, May 2011.

[21] D. Zhang, X. M. Sun, Z. Wu, and W. Wang, "Dissipativity for a class of stochastic nonlinear systems with state-dependent switching," IEEE Trans. Circuits Syst. II Exp. Briefs, vol. 64, no. 1, pp. 86-90, Jan. 2017.

[22] W. M. Xiang and J. Xiao, "Stabilization of switched continuous-time systems with all modes unstable via dwell time switching," Automatica, vol. 50, pp. 940-945, Mar. 2014.

[23] Y. E. Wang, B. Wu, and C. Wu, "Stability and $L_{2}$-gain analysis of switched input delay systems with unstable modes under asynchronous switching," J. Franklin Inst., vol. 354, no. 11, Jul. 2017.

[24] L. F. Zhou and S. Y. Li, "Distributed model predictive control for consensus of sampled-data multi-agent systems with double-integrator dynamics," IET Control Theory Appl., vol. 9, no. 12, Jul. 2015. 